\title{
Advancing Electrolytes Towards Stable Organic Batteries
}

\author{
Yanliang Liang ${ }^{*, a}$ and Yan Yao, ${ }^{*, a}$ \\ ${ }^{a}$ Department of Electrical \& Computer Engineering and Materials Science and Engineering Program, \\ University of Houston, Houston, Texas 77204, USA \\ ${ }^{b}$ Texas Center for Superconductivity, University of Houston, Houston, Texas 77204, USA
}

\begin{abstract}
Organic electrode materials offer virtually infinite resource availability, cost advantages, and some of the highest specific energy for batteries to satisfy the demand for large-scale energy storage. Among the biggest challenges for the practical applications of batteries based on organic electrodes is the dissolution of organic active materials into the electrolyte, which leads to underwhelming cycling stability. This minireview provides an overview of electrolyte advancements to improve the stability of organic batteries. Research efforts on the control of solvent polarity, electrolyte mobility, and exploration of novel electrolyte systems are highlighted.
\end{abstract}

Keywords polymer, small molecule, quasi-solid-state, ionic conductor, composite electrolyte

\section{Introduction}

Batteries powering the world today are constructed predominantly by inorganic active materials consisting of transition metals, which are typically produced via mining and energy-intensive processes. ${ }^{[1]}$ The environmental and cost concerns related to these materials are increasingly worrying as batteries reach into large-scale applications such as grid storage and electric vehicles. ${ }^{[2]}$ Organic electrode materials are emerging as promising alternatives during the last decade: they can be synthesized from inexpensive chemical feedstock and biomass via low-energy processes, and readily recycled. ${ }^{[3]}$ Batteries based on organic electrodes deliver some of the highest specific energies currently known, rivaling other intensively researched technologies based on high-voltage intercalation compounds and even sulfur. ${ }^{[4,5]}$ Despite these benefits, organic batteries have hurdles to overcome before practical application can be realized, among which the compromise between power and effective energy density and that between cycle life and specific energy are particularly noteworthy. This minireview deals with the latter: the cycle life of organic batteries is typically short and mainly attributable to the dissolution of organic active materials to the electrolyte. To battle dissolution, molecular functionalization of small-molecule materials such as polymerization and installation of bulky and/or ionic groups has been extensively investigated as an effective strategy to decrease the solubility. These efforts have been summarized in several recent review articles. ${ }^{[6-9]}$ However, these strategies inevitably decrease the specific capacity of the molecules due to the added weight of redoxinactive auxiliary groups. To keep the specific capacity high, the molecular structure of active materials must stay minimalist, and the task of solubility reduction would need to be fulfilled by the other component: the electrolyte.

Electrolyte advancement has accompanied the development of organic electrode materials as an indispensable tool to optimize electrode performance including cycling stability. Compared with the research interests in the molecular engineering of organic active materials, little attention has been dedicated to the design of electrolytes. Although the choice and function of electrolytes are included in a brief section in some reviews on organic batteries, they deserve more comprehensive summary and insightful analysis. ${ }^{[10,11]}$ Herein, we outline the current knowledge of electrolyte engineering towards more stable organic batteries.

\section{Solvents with low polarity}

By far the most dominant electrolytes for lithium-ion batteries are lithium salts in mixed carbonate solvents. ${ }^{[12]}$ In the typical ethylene carbonate-dimethyl carbonate (EC-DMC) electrolyte, EC with moderate viscosity $(1.9 \mathrm{cP})$ and high polarity $(85.1)$ is used in conjunction with the low-viscosity $(0.59 \mathrm{cP})$ and low-polarity (3.1) solvent DMC (for a summary of physical properties for solvents discussed herein, see Table 1). The balanced viscosity, salt-dissociation capability, and protective layer-forming ability are all important factors to consider in the choice of the electrolyte composition. In the studies on organic batteries, single-component solvents are sometimes used for electrolytes to better control the solution property. Based on the empirical like-dissolves-like rule, one rational strategy to suppress the dissolution of organic materials is to use a solvent that has contrasting properties. One of the solvent properties more easily controllable is polarity, which is routinely characterized by dielectric constant. For this purpose, battery systems based on electrode materials and electrolytes with contrasting polarities have been developed. Examples are the electrolyte choices for salt-form organic molecules: installation of ionic auxiliary groups to small molecules is one of the most established strategies to reduce the solubility of molecules in organic electrolytes because ionic compounds are more polar than typical organic solvents. ${ }^{[13]}$ To achieve the best stability possible for these ionic materials, researchers have used unconventional singlecomponent solvents with low polarity to maximize the polarity difference between the material and the electrolyte. As such, a unique DMC-bis(trifluoromethane)sulfonimide lithium salt (LiTFSI) electrolyte was used for the demonstration of the cycling stability of the ionic molecule pyromellitic diimide dilithium salt (PDI-Li $i_{2}$, molecular structure shown in Figure 1). ${ }^{[13]}$ A small capacity loss of $\sim 5 \%$ was observed over 25 cycles, which stability was rarely observed for small-molecule materials cycled in liquid electrolytes. Similarly, DMC-based electrolytes were used for other ionic molecules including carboxylates ${ }^{[14,15]}$ and sulfonates. ${ }^{[16]}$

\footnotetext{
* E-mail: yliang7@central.uh.edu,yyao4@uh.edu Received September 27, 2017; accepted November 25, 2017.
} 
Table 1 Physical properties of select solvents used in organic batteries

\begin{tabular}{ccc}
\hline Solvent & Dielectric constant & Viscosity/cP \\
\hline DMC & 3.1 & 0.59 \\
DME & 7.2 & 0.59 \\
Butyrolactone & 42 & 1.7 \\
EC & 85.1 & 1.9 \\
PC & 65 & 2.5 \\
Tetraglyme & 7.7 & 3.26 \\
EiPS & 55 & 5.6 \\
EMImTFSI & $\sim 12$ & 21.1 \\
BMImTFSI & $\sim 12$ & 28.7 \\
\hline
\end{tabular}<smiles></smiles>

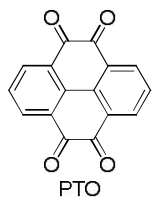<smiles>O=C1c2ccccc2C(=O)c2ccccc21</smiles><smiles>O=C1C(Cl)=C(Cl)C(=O)C(Cl)=C1Cl</smiles><smiles>N#CC(=N)c1ccc(C(C#N)C#N)cc1</smiles><smiles>COC1=C(OC)C(OC)C(=O)C(OC)C1=O</smiles>

TCNQ

TMQ

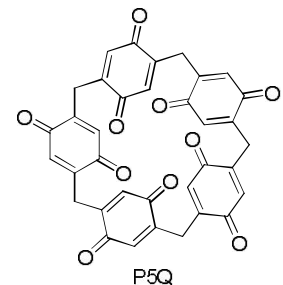

Figure 1 Molecular structures of select organic electrode materials.

Because significant contrast in polarity is necessary for low-polarity electrolytes to help with stability, the usefulness of these electrolytes is limited to highly ionic active materials. For materials without these auxiliary groups, which constitute the majority of organic electrode materials, alternative strategies are necessary.

\section{Highly viscous and concentrated electrolytes}

Most organic electrode materials reported to date are non-ionic at the oxidized state and ionic when reduced. Because the ionicity of the materials constantly changes during charge-discharge, the polarity changes accordingly and spans a large range. Therefore, adjusting the electrolyte polarity alone is usually not enough for performance optimization.

A good example for how solvents affect the cycling stability of typical organic materials is the study of pyrene-4,5,9,10-tetraone (PTO) in electrolytes composed of a wide range of solvents. ${ }^{[17]}$ PTO electrodes underwent very fast capacity decay in electrolytes with low-polarity solvents such as DMC and dimethylethane (DME), losing more than half of their initial capacity within 5 cycles (Figure 2a). In a mixed-solvent electrolyte EC-DMC, the addition of the much more polar EC to DMC improved stability. The best cyclability, however, was observed in imidazolium-based ionic liquid electrolytes, which have polarity in-between DMC and EC. The stability increased in the order 1-butyl-3-methylimidazolium bis(trifluoromethanesulfonyl)imide (BMImTFSI) $>1$-ethyl-3-methylimidazolium bis(trifluoromethanesulfonyl)imide $($ EMImTFSI $)>$ EC-DMC $>$ DMC $\approx$ DME, which did not follow the order of dielectric constant but does align perfectly with that of viscosity (Table 1 ). Therefore, more viscous solvents are beneficial for stabilizing molecular electrode materials. Similarly, Hanyu et al. studied the cycling behavior of small-molecule tetracyanoquinodimethane (TCNQ) electrode in quasi-solid electrolytes containing EMImTFSI, 1-butyl-2-methyl pyrrolidinium bis(trifluoromethanesulfonyl)imide (BMPTFSI), $N, N$-diethyl- $N$-methyl- $N$-(2-methoxyethyl)ammonium bis(trifluoromethanesulfonyl)imide (DEMETFSI), and $N$-methyl- $N$-propylpiperidinium bis(tri- fluoromethanesulfonyl)imide (PP13TFSI) solvents. ${ }^{[18]}$ The rate of capacity fading was recorded in the increasing order of BMPTFSI, PP13TFSI, DEMETFSI, EMImTFSI. This was the reverse order of the viscosity of the ionic liquids. In yet another study, Yokoji subjected 2,5-diiso-propyl-1,4-benzoquinone $\left(i-\mathrm{Pr}_{2}-\mathrm{BQ}\right)$ electrode to three distinct electrolytes: $1.0 \mathrm{M} \mathrm{LiPF}_{6}$ in EC-diethyl carbonate (DEC), $1.0 \mathrm{M} \mathrm{LiN}-\left(\mathrm{SO}_{2} \mathrm{CF}_{3}\right)_{2}$ in ethyl isopropyl sulfone (EiPS), and $2.75 \mathrm{M} \mathrm{LiN}-\left(\mathrm{SO}_{2} \mathrm{CF}_{3}\right)_{2}$ in tetraglyme. ${ }^{[19]}$ The capacity retention of $i$ - $\mathrm{Pr}_{2}$-BQ electrode after 15 cycles in the electrolytes was $30 \%, 54 \%$, and $73 \%$, respectively, making the more viscous solvents EiPS and tetraglyme the favorable options. Although the use of viscous solvents as a strategy to improve stability has not been much investigated, it already appeared to be a common practice among researchers in the evaluation of molecular organic electrodes. Viscous solvents including lactones, higher ethers and carbonates, and ionic liquids are all unconventional lithium electrolyte components but were seen in studies on organic batteries. ${ }^{[20-25]}$
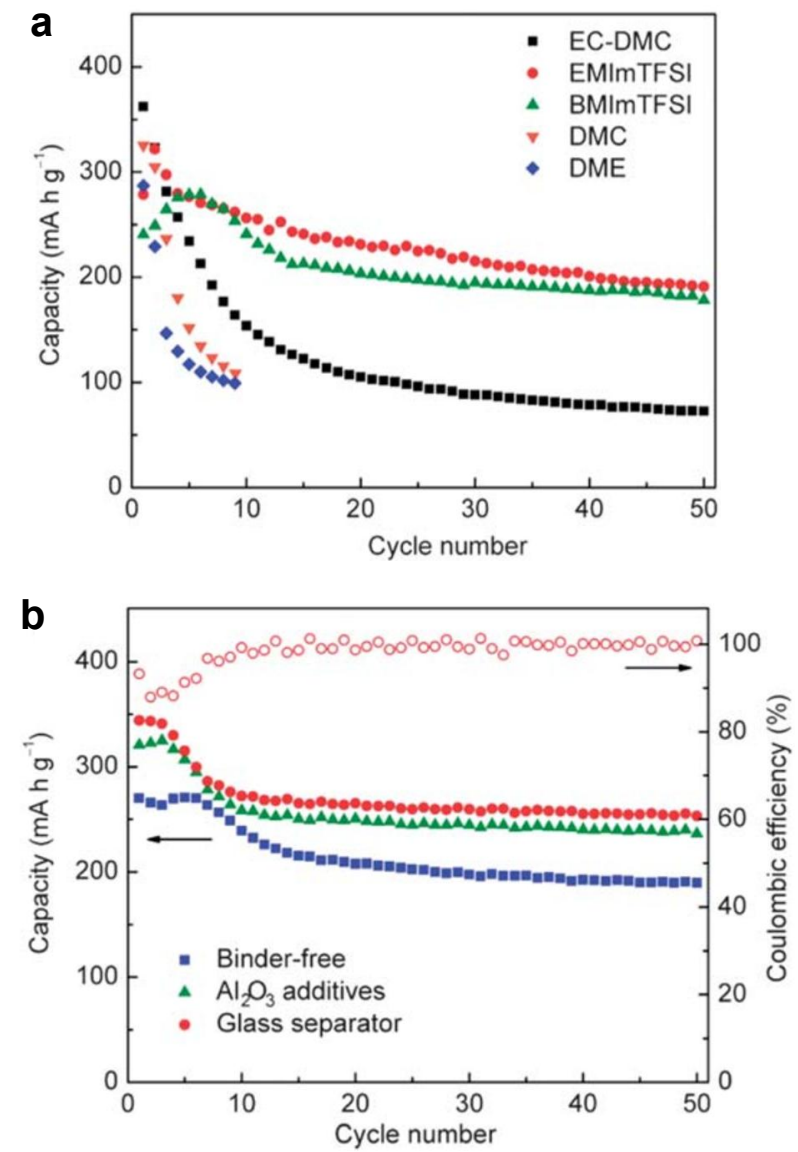

Figure 2 Influence of electrolyte solvent viscosity on the cycling performance of PTO electrodes in nonaqueous lithium cells. (a) Capacity retention in electrolytes based on different solvents with 1 $\mathrm{MLiPF}_{6}$ (for EC-DMC) or LiTFSI (for EMImTFSI, BMImTFSI, $\mathrm{DMC}$, and DME) as the supporting electrolyte. (b) Capacity retention of binder-free electrodes in $1 \mathrm{M}$. $\mathrm{LiClO}_{4}$ inBMImTFSI ( $\mathbf{m}$ PTO-carbon 3:7; $\boldsymbol{\Delta}$ : PTO-carbon- $\mathrm{Al}_{2} \mathrm{O}_{3} 3: 6: 1$; $\bullet$ : PTO-carbon- $\mathrm{Al}_{2} \mathrm{O}_{3}$ $3: 6: 1$, using a glass-fibre separator). ${ }^{[17]}$

More viscous electrolytes can bring about downsides such as low ionic conductivity and unfavorable wetting capability. To counter these limitations, temperatures slightly higher than room temperature have been adopted in some studies. ${ }^{[23]}$ However, using elevated temperatures can become counterproductive because viscosity will decrease as the result, thus negating the advantage of these electrolytes. 
Regulating the composition of the organic electrodes and cell components modifies the wetting properties without increasing temperature. For example, the activation process (capacity increase within initial cycles) observed for PTO electrode in a BMImTFSI electrolyte (Figure 2a) was attributed to the difficulty in electrolyte-electrode wetting, which was effectively alleviated via excluding the ionophobic PVDF and fabricating a binder-free electrode (Figure 2b). ${ }^{[17]}$ Addition of ionophilic $\mathrm{Al}_{2} \mathrm{O}_{3}$ to the electrode and replacing the ionophobic polypropylene separator with glass fiber both further mitigated the activation process.

High viscosity is also achieved by increasing the salt concentration, even with low-viscosity solvents. The rationale behind concentrated $(>1 \mathrm{M})$ electrolytes for organic electrodes is at least two-fold: decreasing the solubility of organic materials and increasing the viscosity of the electrolytes. Guo et al. have studied the solubility of anthraquinone (AQ) in $\mathrm{CF}_{3} \mathrm{SO}_{3} \mathrm{Na}$-tetraglyme electrolytes at salt concentrations of 1-4 M and found continuously reduced AQ dissolution with the increase of electrolyte concentration. ${ }^{[2]}$ In addition, the viscosity of the electrolyte increased by an order of magnitude as the concentration increased from 1 to $4 \mathrm{M}$ (Figure 3a). Both decreased material solubility and increased electrolyte viscosity contributed to longer cycle life: the most concentrated electrolyte, $4 \mathrm{M}$ $\mathrm{CF}_{3} \mathrm{SO}_{3} \mathrm{Na}$ in tetraglyme, afforded the highest reversible specific capacity and capacity retention (Figure $3 b$ ).
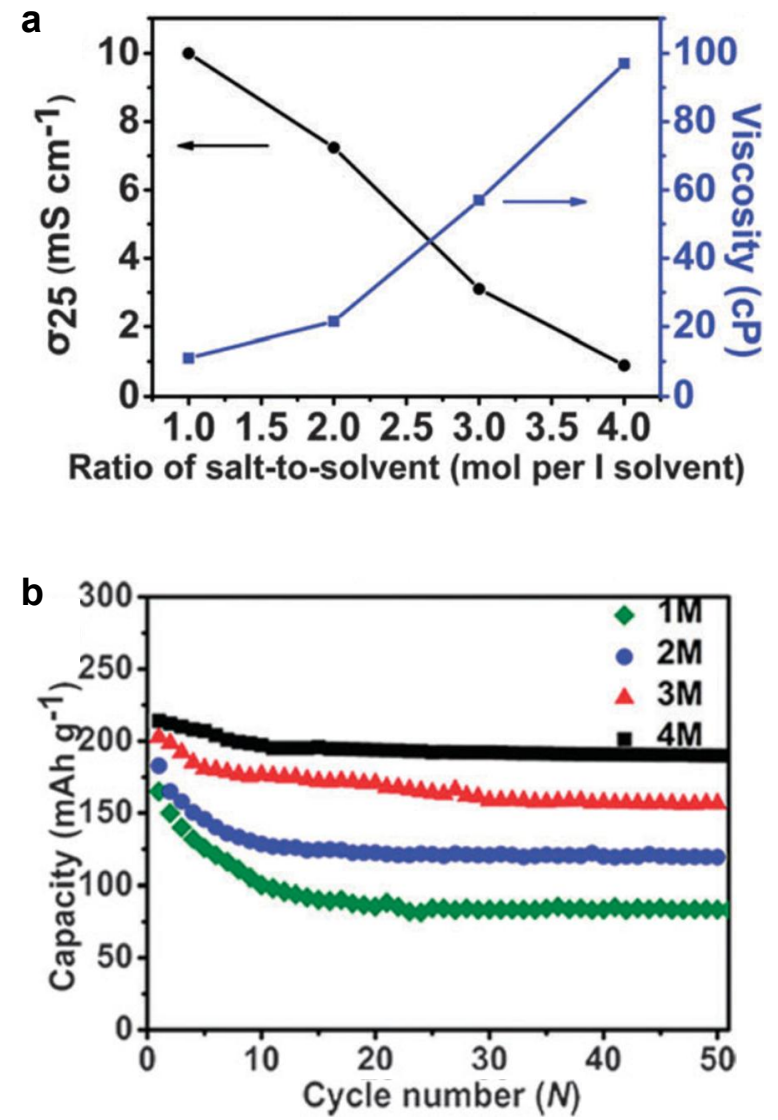

Figure 3 Application of concentrated $\mathrm{CF}_{3} \mathrm{SO}_{3} \mathrm{Na}$-TEGDME electrolyte in nonaqueous sodium cells. (a) Ionic conductivity and viscosity as a function of electrolyte concentration. (b) Cyclability of AQ electrode in these electrolytes. ${ }^{[26]}$

The electrolyte concentration-cell performance relationship can get less straightforward for other cell systems. In a study on AQ in LiTFSI-dioxolane/DME electrolytes, Zhang et al. found that a middling concentration $(2 \mathrm{M})$ gave the highest cycling stability. ${ }^{[27]}$ The concentration of the electrolyte also influenced the voltage profile of
AQ: while a single plateau was observed in 1 and $2 \mathrm{M}$ electrolytes, further increase to 3 and $4 \mathrm{M}$ split the plateau into two. Although the dissolution of AQ was not fully suppressed in these electrolytes, stable cycling can still be achieved by using a $\mathrm{LiNO}_{3}$ additive which formed a protective layer on the surface of the lithium anode and prevented reaction between $\mathrm{AQ}$ and lithium. $\mathrm{Xu}$ et al. reported the dissolution suppression of tannic acid with concentrated (up to $5 \mathrm{M}$ ) LiTFSI-EC/diethylether electrolytes. ${ }^{[28]}$ While $5 \mathrm{M}$ electrolytes gave rise to higher capacity retention than those obtained with less concentrated ones, the moderately concentrated $3 \mathrm{M}$ electrolyte consistently allowed for higher specific capacity at all current densities examined, which was ascribed to the faster electrode kinetics and lower electrolyte resistance in the latter.

Both viscous and concentrated electrolytes slow down the dissolution of organic materials but not stop it. Long-term cycling therefore remains a challenge with these electrolyte systems. Further immobilization of the electrolyte is a natural step going forward, which leads us to (quasi-)solid-state electrolytes.

\section{Quasi-solid-state and all-solid-state electrolytes}

Not content with the delayed material dissolution by modification of liquid electrolytes, Hanyu et al. proposed a quasi-solid-state cell design that drastically reduced the mobility of electrolytes: a layered electrolyte consisting of a $\sim 20 \mu \mathrm{m}$ thick polyethylene oxide (PEO)polyethylene glycol (PEG)-LiTFSI (8:1:1 by weight) layer on the cathode side and a $\sim 400 \mu \mathrm{m}$ thick LiTFSI-EMImTFSI (or BMImTFSI)- $\mathrm{SiO}_{2}(\sim 6 \mathrm{~nm}, 25 \mathrm{~V} \%)$ composite layer on the anode side (Figure 4a). ${ }^{[18]}$ The PEO layer encapsulated the cathode, preventing direct dissolution of the cathode materials TCNQ into the ionic liquid. Because of the reactivity of EMImTFSI with lithium, the lithium

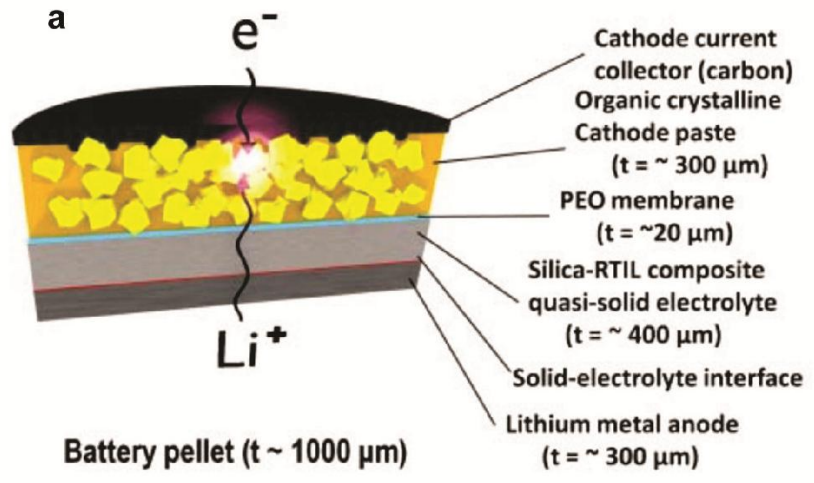

b

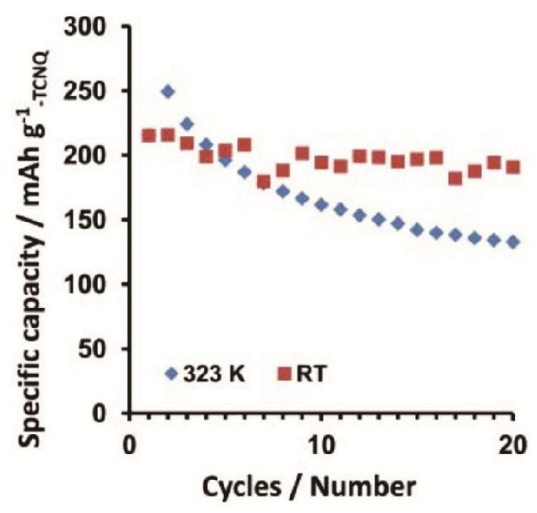

Figure 4 Design and characteristics of a quasi-solid-state organiclithium cell. (a) Cross-section scheme of the quasi-solid-state cell. (b) Cyclability of TCNQ electrode at different temperatures. ${ }^{[18]}$ 
anode was pre-treated with $1 \mathrm{M} \mathrm{LiClO}_{4}$ in $\mathrm{EC} / \mathrm{EMC}$ to form a protective SEI layer. The cells showed decent cycling stability with $70 \%-$ $80 \%$ capacity retention after 100 cycles at $0.2 \mathrm{C}$. This was a significant improvement over a liquid cell with an EC/DEC electrolyte, which retained $\sim 13 \%$ of its initial capacity after 5 cycles. Because of the presence of ionic liquid, cathode dissolution was suppressed but not eliminated in this cell design. It was therefore inevitable that the cell experienced accelerated capacity decay at elevated temperatures (Figure 4b), at which temperatures the ionic liquid became much more mobile and dissolved TCNQ more readily. Ionic liquids with higher viscosity did decrease the decay rate as discussed in previous sections. This quasi-solid-state design had been applied to a variety of organic molecular materials and its effectiveness proves to be general. $^{[29,30]}$

Huang et al. investigated a more typical quasi-solid-state gel electrolyte for calix[4]quinone $(\mathrm{C} 4 \mathrm{Q}) .{ }^{[31]}$ The gel electrolyte consisted of a poly(methacrylate) (PMA)/PEG composite network soaked with $\mathrm{LiClO}_{4}$-dimethylsulfoxide electrolyte. The cells were tested at 283, 293, and $303 \mathrm{~K}$, and enhancement in capacity was observed with the increase in temperature. A higher temperature seemed to lead to a slightly faster decay: the capacity retention at 293 and $303 \mathrm{~K}$ after 20 cycles was $97 \%$ and $94 \%$, respectively.

The above quasi-solid-state attempts obviously show that even with low mobility, the presence of liquid electrolytes is not desirable for long-cycle-life organic batteries. Researchers have therefore explored liquid-free all-solid-state cell designs where polymer and organic crystals serve as solid electrolytes. Note that most of these electrolytes melt at moderately high temperatures. When operated above their melting point, these electrolytes are better described as quasi- instead of all-solid state.

Lécuyer et al. reported a lithium-quinone battery based on a commercial LiTFSI-PEO electrolyte. ${ }^{[32]}$ Cell measurements were solely performed at $100^{\circ} \mathrm{C}$, which was well above the typical melting point of PEO-based Li electrolytes $\left(\sim 60{ }^{\circ} \mathrm{C}\right) .{ }^{[33]}$ The diffusion of the cathode material, tetramethoxy-p-benzoquinone (TMQ), into the electrolyte was therefore not fully inhibited even though no liquid was used. The capacity retention after 40 cycles was $c a .37 \%$ and $79 \%$ at $0.1 \mathrm{C}$ and $1 \mathrm{C}$, respectively, which was still a major improvement from a liquid cell. ${ }^{[34]}$ Despite the material loss from diffusion, TMQ behaved differently from sulfur, which also faced dissolution and related capacity fading. Firstly, the coulombic efficiency of the TMQ cell stayed close to $100 \%$, suggesting no redox shuttle mechanism occurring with the quinone compound. Secondly, the electrolyte-lithium interface appeared rather stable in a lithium-TMQ cell, contrasting the drastically deformed interface in a lithium-sulfur cell (Figures $5 \mathrm{a}$ and $5 \mathrm{~b}$ ). Li et al. fabricated a solid-state cell with a LiTFSI-PEG $\left(M_{\mathrm{w}}=2000\right)$ electrolyte and AQ as the cathode material. ${ }^{[35]}$ The conductivity of the electrolyte was $7.10 \times 10^{-5} \mathrm{~S} \cdot \mathrm{cm}^{-1}$ at 25 ${ }^{\circ} \mathrm{C}$, which was improved to a more adequate $8.21 \times 10^{-4} \mathrm{~S} \cdot \mathrm{cm}^{-1}$ at 65 ${ }^{\circ} \mathrm{C}$. When cycled at $20 \mathrm{~mA} \cdot \mathrm{g}^{-1}$, the cell showed a low capacity of 80 and $180 \mathrm{mAh} \cdot \mathrm{g}^{-1}$ at 35 and $65{ }^{\circ} \mathrm{C}$, respectively (Figure 5c). The higher utilization of active materials at the higher temperature was attributed to the higher ionic conductivity of the electrolyte. The cell showed relatively stable cycling performance at temperatures up to $55{ }^{\circ} \mathrm{C}$, but notable capacity decay at $65{ }^{\circ} \mathrm{C}$ (Figure $5 \mathrm{~d}$ ). Polymer melting may be responsible for the decay: although the melting point of the electrolyte was not provided, the melting point of PEG-2000 was $\mathrm{ca} .50^{\circ} \mathrm{C}$. At $65^{\circ} \mathrm{C}$, the cell likely transitioned from solid-state to quasi-solid-state, resulting in cathode material dissolution.

A polymer electrolyte-based lithium-quinone battery operating at room temperature was reported by Zhu et al. The composite electrolyte consisted of a PMA/PEG polymer blend, $\mathrm{SiO}_{2}(7-10 \mathrm{~nm}, 3 \mathrm{wt} \%)$ filler, and $\mathrm{LiClO}_{4}$ exhibited a maximum ionic conductivity of 0.26 $\mathrm{mS} \cdot \mathrm{cm}^{-1}$ at room temperature (Figure $6 \mathrm{a}$ ). ${ }^{[5]}$ The adequate conductivity allowed the cell to achieve high material utilization $(92 \%)$ without bringing the electrolyte beyond melting temperature, although the
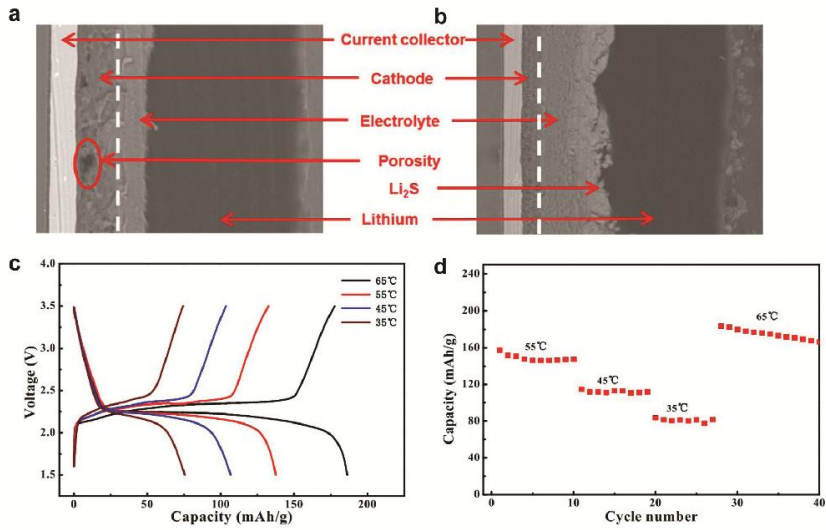

Figure 5 Application of PEO-based electrolytes for organic lithium batteries. ( $a$ and $b$ ) Post-mortem SEM observation of LiTFSI-PEGbased cells with TMQ (a) and sulfur (b) as the active cathode materials. (c) Voltage profile of AQ electrode in a LiTFSI-PEG2000 electrolyte at different temperatures. (d) Cyclability of AQ electrode in the LiTFSI-PEG2000 electrolyte at different temperatures (a and b). ${ }^{[32,35]}$
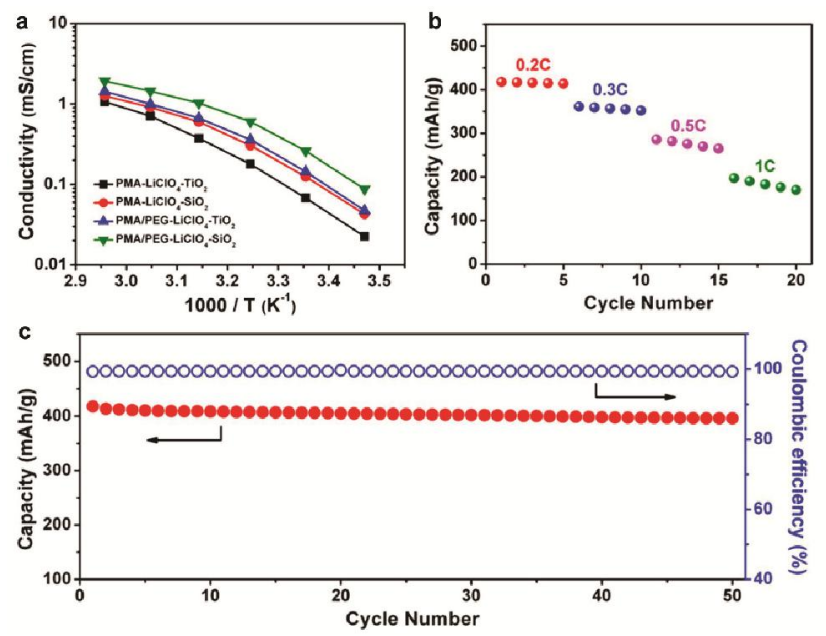

Figure 6 Polymer-ceramic composite electrolytes for organic lithium batteries. (a) Temperature dependence of the ionic conductivity of the electrolytes with $3 \mathrm{wt} \%$ ceramic fillers. (b) Rate capability of P5Q electrode in PMA/PEG-SiO 2 . (c) Cycling stability of P5Q electrode in PMA/PEG-SiO 2 at $0.2 \mathrm{C}^{[5]}$

utilization decreased considerably with the increase of current density (Figure $6 \mathrm{~b}$ ). The cell retained $95 \%$ of its initial capacity after 50 cycles (Figure 6c).

In addition to polymer electrolytes, fast-ionic conductors have been also examined as solid-state electrolytes for organic batteries. Zhu et al. have constructed a $\mathrm{Na}^{+}$conducting electrolyte using succinonitrile as a solid solvent and doped $\mathrm{Na}^{+}$salts $\left(\mathrm{NaClO}_{4}\right.$ and $\left.\mathrm{NaPF}_{6}\right)$ into the organic solid matrix. ${ }^{[36]}$ The electrolytes showed conductivity of up to $\sim 10^{-3} \mathrm{~S} \cdot \mathrm{cm}^{-1}$ at room temperature and stayed solid in the temperature range up to $46{ }^{\circ} \mathrm{C}$. Organic polymers rather than smallmolecule compounds were used as electrode materials, therefore, the effectiveness of the electrolyte in stability improvement could not be directly compared with batteries discussed above. The electrolyte did allow for high-current operation, with a $60 \%$ capacity retention at $800 \mathrm{~mA} \cdot \mathrm{g}^{-1}$, or $c a .4 .5 \mathrm{C}$, though large polarization and electrochemical impedance were observed.

Sato et al. fabricated a bulk-type all- solid-state cell with $\mathrm{LiBH}_{4}$ as the solid-state electrolyte, [6]cyclo-2,7-naphthylene as the cathode active material, and lithium as the anode. ${ }^{[37]}$ The cell saw a capacity 
retention of $95 \%$ after cycling at $\sim 0.6 \mathrm{C}$ at $120{ }^{\circ} \mathrm{C}$ for 65 cycles. The high operating temperature was necessary to induce the highly conducting phase of $\mathrm{LiBH}_{4}{ }^{[38]}$ The potential difference between charge and discharge increased from $\sim 90 \mathrm{mV}$ at $\sim 0.1 \mathrm{C}$ to $\sim 130 \mathrm{mV}$ at $\sim 0.6$ $\mathrm{C}$, indicating decent kinetics. Because of the high reducing capability of complex hydrides, these electrolytes are likely only suitable for use in conjunction with low- potential electrode materials.

Overall, all-solid-state electrolytes have enabled some of the most stable cycling performance for small-molecule organic batteries, though they are yet to strike a good balance among mechanical/ chemical stability, ionic conductivity, and operating temperature. Alternative electrolyte systems are still highly desired, and much longer cycle life is needed for practical applications.

\section{Aqueous electrolytes}

Electrolyte systems discussed above are all based on metal-ionconducting nonaqueous electrolytes. Aqueous electrolytes, notably the proton-conducting acidic electrolytes, have enabled some unexpectedly long cycle life for small-molecule materials albeit liquid electrolytes. PTO exhibited very stable cycling stability in a $4.4 \mathrm{M}$ $\mathrm{H}_{2} \mathrm{SO}_{4}$ electrolyte, showing no obvious capacity decay after $>1500$ cycles/1200 h at $2 \mathrm{C}$ (Figure 7a). ${ }^{[39]}$ Such stability was particularly noteworthy when considering that the electrolyte is fully fluid with a relatively low viscosity of $c a .1 .3 \mathrm{cP}$. In contrast, neutral aqueous electrolytes were about as dissolving as any common non-aqueous electrolytes: dissolution of PTO in a neutral lithium electrolyte (2.5 $\mathrm{M} \mathrm{Li}_{2} \mathrm{SO}_{4}$ ) happened so fast that only $20 \%$ capacity could be retained at the second cycle, and a polymerized version of the compound, PPTO, was necessary to achieve stable cycling (Figure 7b). The stability enhancement by acidic electrolytes seemed to be generally applicable. A tetrachloro- $p$-benzoquinone electrode lost $<5 \%$ capacity after 50 cycles in a $3 \mathrm{M} \mathrm{H}_{2} \mathrm{SO}_{4}$ electrolyte, ${ }^{[40]}$ which contrasted drastically with the $>70 \%$ capacity loss within 20 cycles in a carbo-

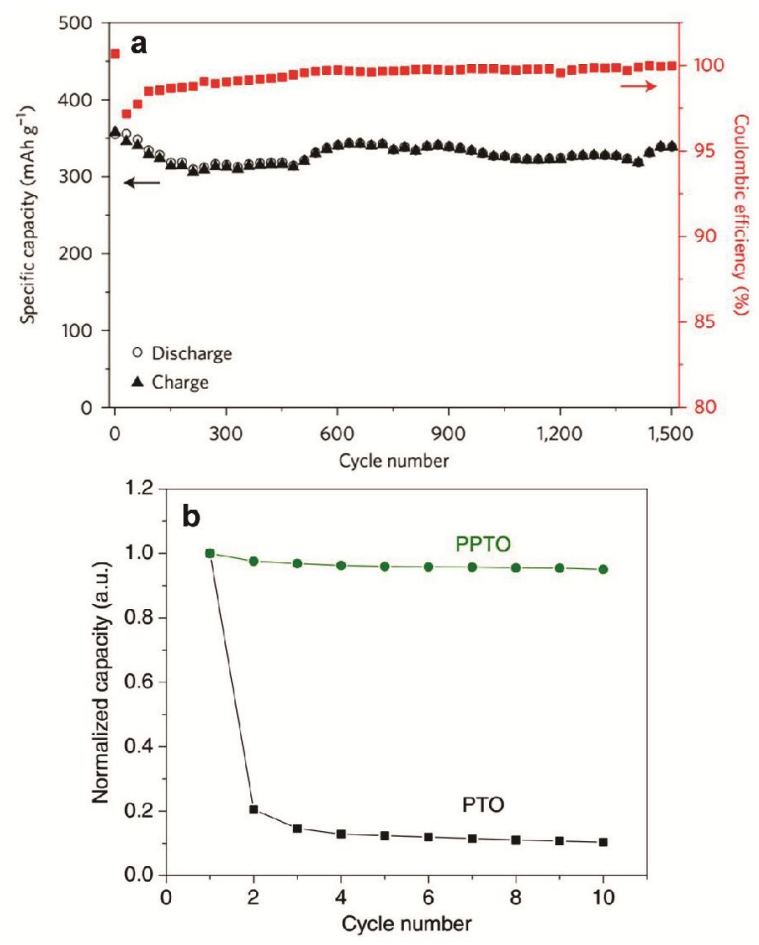

Figure 7 Improving the cyclability organic electrodes by the use of acidic electrolytes. (a) Cyclability of PTO in $4.4 \mathrm{M} \mathrm{H}_{2} \mathrm{SO}_{4}$. (b) Cyclability of PTO and PPTO in a neutral lithium electrolyte ${ }^{[39]}$ nate-based electrolyte despite the use of high-surface-area carbon as an adsorbent. ${ }^{[41]}$ The plausibility to use aqueous electrolytes to realize highly stable organic batteries is promising in that aqueous batteries offer an intrinsically safe solution to large-scale energy storage applications such as electric vehicles and grids.

\section{Conclusions}

The stability of organic molecules-based batteries has seen continuous improvement through the optimization of electrolyte polarity, viscosity, mobility, and careful choice of solid-state and aqueous electrolytes. Recent advancements in polymer, superionic, and solvent-in-salt electrolytes are certainly useful tools to continue the momentum. ${ }^{[42-44]}$ The advances in electrolytes, combined with the increasing understanding in molecular design towards stability, are bringing high-energy organic batteries closer to reality.

\section{Acknowledgement}

This work was supported by the U.S. Department of Energy's Office of Energy Efficiency and Renewable Energy (EERE), as part of the Battery 500 Consortium, Award Number DE-EE0008234.

\section{References}

[1] Armand, M.; Tarascon, J. M. Nature 2008, 451, 652.

[2] Vesborg, P. C. K.; Jaramillo, T. F. RSC Adv. 2012, 2, 7933

[3] Poizot, P.; Dolhem, F. Energy Environ. Sci. 2011, 4, 2003.

[4] Chen, H.; Armand, M.; Demailly, G.; Dolhem, F.; Poizot, P.; Tarascon, J.-M. ChemSusChem 2008, 1, 348.

[5] Zhu, Z.; Hong, M.; Guo, D.; Shi, J.; Tao, Z.; Chen, J. J. Am. Chem. Soc. 2014, 136, 16461.

[6] Liang, Y.; Tao, Z.; Chen, J. Adv. Energy Mater. 2012, 2, 742.

[7] Song, Z.; Zhou, H. Energy Environ. Sci. 2013, 6, 2280

[8] Muench, S.; Wild, A.; Friebe, C.; Häupler, B.; Janoschka, T.; Schubert, U. S. Chem. Rev. 2016, 116, 9438

[9] Zhao, Q.; Zhu, Z.; Chen, J. Adv. Mater. 2017, 1607007.

[10] Zhao, Q.; Guo, C.; Lu, Y.; Liu, L.; Liang, J.; Chen, J. Ind. Eng. Chem. Res. 2016, 55, 5795 .

[11] Häupler, B.; Wild, A.; Schubert, U. S. Adv. Energy Mater. 2015, 5, 1402034.

[12] Xu, K. Chem. Rev. 2014, 114, 11503.

[13] Renault, S.; Geng, J.; Dolhem, F.; Poizot, P. Chem. Commun. 2011, 47, 2414.

[14] Renault, S.; Brandell, D.; Gustafsson, T.; Edstrom, K. Chem. Commun 2013, 49, 1945.

[15] Renault, S.; Mihali, V. A.; Brandell, D. Electrochem. Commun. 2013, 34, 174.

[16] Wan, W.; Lee, H.; Yu, X.; Wang, C.; Nam, K.-W.; Yang, X.-Q.; Zhou, H. RSC Adv. 2014, 4, 19878.

[17] Liang, Y.; Zhang, P.; Chen, J. Chem. Sci. 2013, 4, 1330.

[18] Hanyu, Y.; Honma, I. Sci. Rep. 2012, 2, 453.

[19] Takato, Y.; Yuki, K.; Shun, S.; Norihiko, M.; Masaharu, S.; Hiroshi, M. Chem. Lett. 2015, 44, 1726.

[20] Yao, M.; Senoh, H.; Araki, M.; Sakai, T.; Yasuda, K. ECS Trans. 2010 , 28,3 .

[21] Yao, M.; Senoh, H.; Yamazaki, S.-I.; Siroma, Z.; Sakai, T.; Yasuda, K. J. Power Sources 2010, 195, 8336.

[22] Yao, M.; Senoh, H.; Sakai, T.; Kiyobayashi, T. Int. J. Electrochem. Sci. 2011, 6, 2905.

[23] Nokami, T.; Matsuo, T.; Inatomi, Y.; Hojo, N.; Tsukagoshi, T.; Yoshizawa, H.; Shimizu, A.; Kuramoto, H.; Komae, K.; Tsuyama, H.; Yoshida, J.-i. J. Am. Chem. Soc. 2012, 134, 19694.

[24] Yao, M.; Ando, H.; Kiyobayashi, T. Energy Procedia 2013, 34, 880.

[25] Yao, M.; Kuratani, K.; Kojima, T.; Takeichi, N.; Senoh, H.; Kiyobayashi, T. Sci. Rep. 2014, 4, 3650. 
[26] Guo, C.; Zhang, K.; Zhao, Q.; Pei, L.; Chen, J. Chem. Commun. 2015, 51, 10244 .

[27] Zhang, K.; Guo, C.; Zhao, Q.; Niu, Z.; Chen, J. Adv. Sci. 2015, 2, 1500018.

[28] Xu, Z.; Ye, H.; Li, H.; Xu, Y.; Wang, C.; Yin, J.; Zhu, H. ACS Omega 2017, 2, 1273.

[29] Hanyu, Y.; Ganbe, Y.; Honma, I. J. Power Sources 2013, 221, 186.

[30] Hanyu, Y.; Sugimoto, T.; Ganbe, Y.; Masuda, A.; Honma, I. J. Electrochem. Soc. 2014, 161, A6.

[31] Huang, W.; Zhu, Z.; Wang, L.; Wang, S.; Li, H.; Tao, Z.; Shi, J.; Guan, L.; Chen, J. Angew. Chem., Int. Ed. 2013, 52, 9162.

[32] Lécuyer, M.; Gaubicher, J.; Barrès, A.-L.; Dolhem, F.; Deschamps, M.; Guyomard, D.; Poizot, P. Electrochem. Commun. 2015, 55, 22.

[33] Croce, F.; Appetecchi, G. B.; Persi, L.; Scrosati, B. Nature 1998, 394, 456.

[34] Barrès, A.-L.; Geng, J.; Bonnard, G.; Renault, S.; Gottis, S.; Mentré, O.; Frayret, C.; Dolhem, F.; Poizot, P. Chem. Eur. J. 2012, 18, 8800.

[35] Li, W.; Chen, L.; Sun, Y.; Wang, C.; Wang, Y.; Xia, Y. Solid State Ionics 2017, 300, 114 .
[36] Zhu, X.; Zhao, R.; Deng, W.; Ai, X.; Yang, H.; Cao, Y. Electrochim. Acta $\mathbf{2 0 1 5}, 178,55$

[37] Sato, S.; Unemoto, A.; Ikeda, T.; Orimo, S.-I.; Isobe, H. Small 2016, 12, 3381.

[38] Matsuo, M.; Orimo, S.-I. Adv. Energy Mater. 2011, $1,161$.

[39] Liang, Y.; Jing, Y.; Gheytani, S.; Lee, K.-Y.; Liu, P.; Facchetti, A.; Yao, Y. Nat. Mater. 2017, 16, 841.

[40] Alt, H.; Binder, H.; Köhling, A.; Sandstede, G. Electrochim. Acta 1972, 17,873 .

[41] Kim, H.; Kwon, J. E.; Lee, B.; Hong, J.; Lee, M.; Park, S. Y.; Kang, K. Chem. Mater. 2015, 27, 7258 .

[42] Miller, T. F.; Wang, Z.-G.; Coates, G. W.; Balsara, N. P. Acc. Chem. Res. 2017, 50, 590.

[43] Manthiram, A.; Yu, X.; Wang, S. Nat. Rev. Mater. 2017, $2,16103$.

[44] Suo, L.; Borodin, O.; Gao, T.; Olguin, M.; Ho, J.; Fan, X.; Luo, C.; Wang, C.; Xu, K. Science 2015, 350, 938. 